\title{
Actual Call Connection Time Characterization for Wireless Mobile Networks Under a General Channel Allocation Scheme
}

\author{
Wei Li, Member, IEEE, Yuguang Fang, Senior Member, IEEE, and Robert R. Henry, Senior Member, IEEE
}

\begin{abstract}
Actual call connection time (ACCT) is the total time that a mobile user engages in communications over a wireless network during a call connection. Due to limited network resources of wireless mobile networks, a call connection may be prematurely disconnected and the ACCT for the call in general may not be the same as the requested call connection time (RCCT). The ACCT depends not only on the RCCT, but also on the network resource allocation scheme and network traffic. In this paper, we characterize the ACCT and related performance metrics for wireless mobile networks under a newly proposed general channel allocation scheme. This scheme generalizes the nonprioritized scheme, the reserved channel scheme, the queueing priority scheme and the subrating scheme in such a way as to reduce the blocking probability of the handoff calls while keeping the ACCT as long as possible. Explicit formulae for the distribution and the expectation of the ACCT are obtained. The call completion probability, the call drop probability, and the average actual call connection times for both the complete calls and the incomplete calls are derived. The results can form the basis for designing better billing rate schemes by differentiating incomplete calls and complete calls.
\end{abstract}

Index Terms-Actual call connection time, blocking probability, call dropping probability, handoff, mobility, personal communication service, wireless networks.

\section{INTRODUCTION}

$\mathbf{T}$ HE NEXT-generation wireless mobile networks promise to provide a wide variety of services such as voice, data, and multimedia to users on the move. Mobile users can make a phone call as in wired telephony, or make a connection to retrieve information messages such as email or stock information, even make a connection to surf the Internet, or to do business over the Internet (electronic commerce or mobile commerce). Due to the limited resources (time, frequency, and code)

Manuscript received September 20, 2000; revised August 10, 2001; accepted August 30, 2001. The editor coordinating the review of this paper and approving it for publication is B. Li. The work of W. Li was supported in part by the Louisiana Board of Regents Support Fund under Contract LEQSF(2000-03)-RD-A-40. The work of Y. Fang was supported in part by the National Science Foundation Faculty Early Career Award under Grant ANI 0093241 and in part by the Office of Naval Research Young Investigator Award under Grant N000140210464.

W. Li was with the Department of Electrical and Computer Engineering, University of Louisiana at Lafayette, Lafayette, LA 70504-3890 USA. He is now with the Department of Electrical Engineering and Computer Science, University of Toledo, Toledo, OH 43606-3390 USA (e-mail: wli@eecs.utoledo.edu).

Y. Fang is with the Department of Electrical and Computer Engineering, University of Florida, Gainesville, FL 32611 USA (e-mail: fang@ece.ufl.edu).

R. R. Henry is with the Department of Electrical and Computer Engineering, University of Louisiana, Lafayette, LA 70504, USA (e-mail: henry@louisiana.edu).

Digital Object Identifier 10.1109/TWC.2002.804191 of such networks, ongoing call connections may be disrupted or dropped, leading detrimental effect on customer satisfaction. In order to address this issue, it seems a good idea to apply different charging rates to the complete call connections and incomplete call connections([8], [17]). Thus, the actual call connection time (ACCT), the time a mobile user actually uses the network services during a call connection, and the ACCT for both incomplete call connections and complete call connections will be important quantities for such design of billing schemes.

Wireless networks such as personal communication service (PCS) networks have the service coverage area populated with base stations, which are interconnected with either public telephone networks or packet switching networks, the backbone networks for providing services to mobile users. Each base station is responsible for relaying service to mobile users in its area, called a cell. In each cell, there are usually two classes of call traffic: new calls and handoff calls. A new call is the call connection which initiates in the current cell, while a handoff call is the call connection which initiates in another cell and is handed over to the current cell. When a new call originates in the current cell, if an unused channel is available, it will be assigned for the communication between the mobile user and the base station and a new call is accepted for service. If a channel cannot be assigned to the new call, based on the channel allocation scheme, the new call may be blocked and cleared from the system, in which case, the ACCT for this call is, therefore, zero. When a new call gains a channel, it will keep the channel either until it is completed in the cell or until the mobile moves out of the cell (we do not consider the channel reassignment in this paper). If the call is completed in the cell, the ACCT is equal to the requested call connection time (RCCT). If the mobile user does not finish the service in the originating cell, it will move out of the cell and attempt to gain service (a channel) in another cell. If the call does not acquire resource (either buffers or a channel), it will be forced to terminate. In this case, the ACCT will be the time from the start of the call to the time that the call is terminated prematurely. If the handoff is successful, then the call will continue in the new cell, and the ACCT will accumulate and the same procedure repeats.

As we observe from the above discussion, the ACCT depends on the channel (resource) allocation scheme used for handling new calls and handoff calls. The following is a brief review of channel allocation schemes found in the current literature.

- Nonprioritized Scheme (NPS): New calls and handoff calls are treated equally. Thus, either a new call or a handoff call will be served as long as there is a channel available in the cell. This scheme has been employed by the typical radio 


\begin{tabular}{c|c|c|c|c}
\hline \multicolumn{2}{c|}{ State Variables } & \multicolumn{2}{c}{ Events/Action } \\
\hline \# of calls (i) & $\begin{array}{c}\text { \# of calls with } \\
\text { split channels }\end{array}$ & $\begin{array}{c}\text { \# of calls in } \\
\text { the buffer }\end{array}$ & New call arrival & Handoff call arrival \\
\hline $0 \leq i<K-C$ & 0 & 0 & Accepted (full rate) & Accepted (full rate) \\
\hline$K-C \leq i<K$ & 0 & 0 & Blocked & Accepted (full rate) \\
\hline$K \leq i<K+C$ & $2(i-K)$ & 0 & Blocked & Accepted (split rate) \\
\hline$K+C \leq i<K+C+N$ & $2 C$ & $i-(K+C)$ & Blocked & Buffered \\
\hline$i=K+C+N$ & $2 C$ & $N$ & Blocked & Blocked \\
\hline
\end{tabular}

Fig. 1. Proposed GCAS state machine description.

technologies proposed for PCS systems operated around $2 \mathrm{GHz}$ [20].

- Reserved Channel Scheme (RCS) (also called guard channel or cutoff priority scheme): In this scheme, $m(0 \leq m<M)$ out of a total of $M$ channels in each cell are reserved for handoff calls. Thus, a new call can gain a free channel only if at least $m+1$ channels in the cell are free. A handoff call will always gain a channel as long as there is any channel available. Notice that only the number of channels, not individual channels, are reserved. The overall effect of this scheme is that new calls will be blocked if at least $M-m$ channels are in use, while handoff calls will always be served if there are idle channels in the cell [18], [23].

- Queueing Priority Scheme (QPS): New calls are blocked when there are no free channels available in the cell. When the handoff calls find that all channels are occupied, they are queued according to various queueing disciplines and wait for an occupied channel to be released [20], [16].

- Subrating Scheme (SRS): In this scheme, certain channels are allowed to be temporarily divided into two channels at half of the original rate to accommodate handoff calls. This SRS will be activated when all channels are occupied at the moment of a handover call arrival. When a subrated channel is released, it forms into an original full-rated channel by combining with another subrated channel [17], [21].

In this paper, we propose the following more general channel allocation scheme, which combines the idea of a guard channel, queueing and SRSs, which is described as follows.

1) Each cell consists of $K$ channels of rate $l \mathrm{~kb} / \mathrm{s}$ and a buffer with size of $N$ for handoff calls only. $C(0 \leq C \leq K)$ channels in each cell are reserved for handoff calls and each of them can be split into two channels with different rates $l_{1} \mathrm{~kb} / \mathrm{s}$ and $l_{2} \mathrm{~kb} / \mathrm{s}$, respectively, when a handoff call arrives and finds $i(K \leq i<K+C)$ calls in the cell.

2) A new call will gain a full rate channel for service when it arrives and finds there are only $i(0 \leq i<K-C)$ calls in the cell. Otherwise, the new call will be blocked and cleared from the system.

3) A handoff call will also gain a full rate channel for service when it arrives and finds the total number of calls in the cell is less than $K$. However, if a handoff call finds all channels are busy upon its arrival and the number of split channels in the cell is less than $C$, one of the full rate channel will be split into two split channels, one keeping the original call and the other one being assigned for the coming handoff call. If the number of split channels is $C$ upon the handoff call's arrival, the call will be placed on hold until the time at which either it gains a channel for service, or it disconnects from the system because of the user's impatience, or the mobile moves out of the current cell. If a handoff call finds all positions in the handoff queue are occupied when it comes, it will be forced to terminate and is cleared from the system.

A state machine description of this proposed general channel allocation scheme is given in Fig. 1.

The purpose of our proposed scheme is to incorporate the commonly used schemes of NPS, RCS, QPS, and SRS in such a way as to cast the channel allocation scheme in a unified framework, thus, we can reduce the blocking probability of handoff calls while keeping the ACCT as long as possible. Our focus of this paper is to find an unifying expression of the average ACCT for a call connection, for a complete call connection and for an incomplete call connection, respectively, under the general channel allocation scheme. Based on the average ACCT for a complete call and an incomplete call, the operating companies can design more reasonable charging scheme as already shown in some of the co-authors' work [8], [18]: incomplete calls are charged less for better customer care. Operating companies can, based on the ACCT for complete calls and incomplete calls, design a scheme to minimize the overall revenue loss while taking care of the customers' satisfaction by charging complete calls higher rate and incomplete call lower rate. Moreover, it is intuitive that the blocked handoff calls and the incomplete calls can significantly be reduced because the general channel allocation scheme (GCAS) possesses all the advantages for the handoff calls in the four schemes. In fact, this is the main advantage of our scheme if one compares it with other four schemes. Furthermore, the ACCT of the new scheme tends to be longer than anyone obtained based on the four schemes because of more chances for handoff calls to gain access, due to incorporation of queueing and subrating ideas in our new scheme. In fact, the new scheme are expected to include all advantages of the four schemes from viewpoint of billing strategies and the minimization of the blocked handoff calls. One disadvantage of this general scheme is that some interested results, such as ACCT for any call, the ACCT for a complete call and for an incomplete call, may be too difficult to achieve analytically under more general assumptions on the cell traffic and holding time distributions due to the complicated operational structure of the channel allocation scheme. This paper, however, is taking the first step to obtain the closed form results for the ACCT for a call, the ACCT for an incomplete call and a complete call. It is our belief that our results can be used to develop enhanced billing schemes, which will provide for increased satisfaction for the network service provider and the customers.

This paper is organized as follows. In Section II, we list assumptions and notations used in this paper. We then present analytical characterization of the ACCT in Section III. The 
analysis for the ACCT for incomplete calls and complete calls are given in Section IV. In Section V, we present results for the cases when the call processes are independent call arrival processes, dependent call arrival processes, and the correlated call arrival processes, respectively. We conclude the paper in Section VI.

\section{ASSUMPTION AND NOTATION}

In this section, we provide a detailed description for GCAS and notation we use in this paper. Relationships between this scheme and the previous schemes are also discussed. The basic assumption of the GCAS, except those already described in Section I, are as follows.

1) The wireless mobile network here is assumed to be homogeneous (see Remark 1 and paper [25]), i.e., the underlying processes and parameters for all cells within the cellular networks are statistically identical.

2) Each cell consists of $K$ channels and a buffer of size $N$ for handoff calls only. $C(0 \leq C \leq K)$ channels in each cell can be split into two channels with rates $l_{1}-\mathrm{kb} / \mathrm{s}$ and $l_{2}-\mathrm{kb} / \mathrm{s}$, respectively, when a handoff call arrives and find $i(K \leq i<K+C)$ calls in the cell. The original rate for each channel is $l-\mathrm{kb} / \mathrm{s}$, which is the sum of $l_{1}-\mathrm{kb} / \mathrm{s}$ and $l_{2}-\mathrm{kb} / \mathrm{s}$.

3) Each handoff call arriving at the cell and having to wait in the handoff area has a patience time. If the virtual waiting time (i.e., the time which the handoff call would have to wait until service) exceeds the patience time, the handoff call departs from the system and is lost by impatience. The patience times of the handoff calls are assumed to be independent and identically distributed (i.i.d.) with exponential distribution and with an expectation 1/d. See [1]-[4], [12], and [27] for more application of the impatience phenomena in various systems.

4) The RCCT, $\mathbf{H}$, of a call is defined as the call duration without forced termination. This time is also called the call holding time ([6]) or the unencumbered session time ([23]). As usually [14], [21], it is assumed to be exponentially distributed with mean $1 / h$ on the original channel with rate $l-k b / s$ and exponentially distributed with mean $1 / h_{i}(i=1,2)$ on the split channels with rate $l_{i}-k b / s$, where $\max \left\{h_{1}, h_{2}\right\} \leq h \leq h_{1}+h_{2}$.

5) The cell residence time, $R$, of each mobile is defined as the interval that a mobile user stays in a cell and is assumed to be exponential distribution with rate $r$. Usually, it depends on the mobile velocity and direction as well as the shape of the cells. Some reasons for assumptions of the exponential distribution for RCCT and cell residence time are listed in Remark 2.

6) Handoff area has been defined as the area in which the average received power level from the base station is between the handoff threshold level and the receiver threshold level [13]. Here, the handoff threshold is set at the point where the power received from the BS of a neighboring cell site has started to exceed the power received from the current BS by a certain amount and/or for a certain time and the receiver threshold is the point at which the received power from the BS is at the minimum acceptable level [26]. The handoff area residence time is a random variable and usually also depends on the mobile velocity and direction as well as the shape of the cells. We use the mean of this variable to approximately produce an exponential time with same mean, which is denoted by $1 / a$. This time has the relationship with the queueing time. In fact, based on our scheme, the queueing time is the minimum of this variable and the patient time.

7) All random variables above are assumed to be independent each other.

It is worthwhile to point out that if the arrival processes of the new calls and handoff calls are independent each other and are Poisson processes, our general scheme will reduce to some popular schemes as follows.

- If $C=0$ and $N=0$, the scheme is the same as the NPS.

- If $C<K, N=0$, and $h_{1}=h_{2}=h$, the scheme is equivalent to the RCS, in which the total number of channels in a cell is $M=K+C$ and number of reserved channels for handoff calls is $m=2 C$.

- If $C=0$, the scheme is the same as the QPS.

- If $h_{1}=h_{2}=h / 2$ and $N=0$, the scheme becomes the SRS.

Remark 1: The homogeneity assumption implies that the structures of all cells within the PCS networks are statistically identical. This is possible in the reality if the network areas are almost similar, for example, if there are no high mountains, no large lakes. Most performance studies in cellular networks commonly invoke this assumption in order to obtain tractable analytical results. This is particularly true for PCS networks because the cell size is reduced significantly. The main technical difficulty for the nonhomogeneous case is the lack of realistic modeling of the interactions of the call traffic among cells (particularly the neighboring cells). To the authors' knowledge, there is no good model for nonhomogeneous cellular networks in the current literature.

Remark 2: Since we are only handling call connection level performance, the requested call connection time corresponds to the regular call holding time in wired telephone systems for voice services and session time for data services in computer systems. The exponential model has been shown to appropriate for call holding time in telephone systems in the past. In [24], the authors also observed that some TCP session time, such as for remote login, is well modeled by exponential distribution. Moreover, we use the exponential assumption mostly because we want to derive analytical results as commonly done in the current literature. The focus of this paper is not on whether these assumptions are appropriate or not. However, for more general discussions on the validity of such assumptions, please refer to some papers by one of the coauthors (see [6], [7], and references therein). Under NPS and RCS schemes, the exponential distribution has been extended to the more general model (such as hyper-Erlang) for call performance evaluation ([5], [9], [18]).

Remark 3: It is also noteworthy to point out that there is no specific assumption on the call arrival processes in the GCAS. Potential processes such as independent call arrival processes, 
dependent call arrival processes or correlated call processes can be used to model the cell traffic.

\section{Actual Call Connection Time (ACCT)}

The ACCT per call is the total time that the mobile user spends in the network. It depends not only on the customer's requirement but also on the network structure (including the channel schemes). It will be of great interest to know the distribution of the ACCT as described in the introduction. In the past, the ACCT has been studied for the cases when no queueing is allowed [8], [9], [18]. In [18], this measure is called actual call holding time for the RCS. We study the ACCT under the general channel allocation scheme.

As we pointed out in the RCS [18], the ACCT cannot be greater than RCCT because there is no buffer in the cell. However, in the current general scheme, we observe that: 1) the ACCT can be greater than the RCCT and 2) the call may be incomplete even if the ACCT is greater than the RCCT. These are possible because handoff calls can wait in the handoff area during the handoff process and the actual call connection time contains not only the time in service but also the possible waiting time.

Let $P(X<x)$ denote the distribution function of the ACCT, say $X$, of a typical call. We find this distribution can be clearly derived in terms of the unit step function. Based on the above observation, if we introduce four events $A, B_{1}, B_{2}$, and $C$ as:

- $A$ : the new call is blocked; and $\bar{A}$ means the event that the new call is not blocked;

- $C: A C C T \geq R C C T$ and the call is a complete call;

- $B_{1}: A C C T \geq R C C T$ and the call is an incomplete call;

- $B_{2}: A C C T<R C C T$ (the call should be an incomplete call);

we have

$$
\begin{aligned}
P(X<x)=P(X< & x, A)+P(X<x, \bar{A} C) \\
& +P\left(X<x, \bar{A} B_{1}\right)+P\left(X<x, \bar{A} B_{2}\right) .
\end{aligned}
$$

In order to characterize $P(X<x, A), P(X<x, \bar{A} C)$, $P\left(X<x, \bar{A} B_{1}\right)$, and $P\left(X<x, \bar{A} B_{2}\right)$, we must know the new call blocking probability and the forced termination probability. For this purpose, we introduce the following notation and some basic results.

- $p_{0}$ : the new call blocking probability, i.e., the probability that a new call is blocked and cleared from the system in the originating cell due to the lack of an available channel.

- $p_{f}$ : the probability that a handoff call is forced to terminate in a handoff area, i.e., the probability that a handoff call is blocked and cleared from the system in the handoff area, due to the possibilities that either the buffer being full or its waiting time in the buffer exceeding its maximum waiting time.
- $\phi_{k}(k=0,1, \ldots, N-1)$ : the interval from the epoch that the call arrives and finds $K+C+k$ calls already in the cell, to the epoch that one of the $K+C+k$ call leaves the cell.

- $\tau$ : the maximum queueing time of a handoff call in the buffer, i.e., the minimum of the call's handoff area residence time and the call's patience time in the buffer.

- $b_{i}(i=1,2, \ldots, N+K+L)$ are given by the equation at the bottom of the page, where $\mu_{0}=h+r, \mu_{1}=$ $h_{1}+h_{2}+2 r$ and $\mu_{2}=d+a$. It is straightforward to show that $b_{i}$ is in fact the departure rate when there are $i$ calls in the cell.

Based on the exponential assumptions and a direct probabilistic analysis, we know that $\phi_{k}$ is exponentially distributed with expectation $1 / b_{K+C+k}$ and that $\tau$, the minimum of the patience time and the handoff area residence time, is also exponentially distributed and has an expected value $1 / \mu_{2}$. Therefore, the probability that a handoff call is forced to terminate in a handoff area is given by

$$
\begin{aligned}
p_{f} & =\sum_{j=0}^{C+K+N} P(\text { the arriving handoff call finds } j \text { calls } \\
& \text { in the cell and the call is finally forced to terminate }) \\
& =\bar{\pi}_{K+C+N}+\sum_{j=0}^{N-1} \bar{\pi}_{K+C+j} P\left(\tau<\phi_{0}+\cdots+\phi_{j}\right) \\
& =\bar{\pi}_{K+C+N}+\sum_{j=0}^{N-1} \bar{\pi}_{K+C+j} \frac{(j+1) \mu_{2}}{b_{K+C+j+1}}
\end{aligned}
$$

and the new call blocking probability is given by

$$
p_{0}=\sum_{j=K}^{K+C+N} \tilde{\pi}_{j}
$$

where $\bar{\pi}_{n}\left(\tilde{\pi}_{n}\right)$ denotes the steady-state probability that an arriving handoff (new) call finds there are already $n$ calls in the cell and $\sum_{j=0}^{-1} y_{j}=0$. These probabilities closely depend on the arrival processes and their explicit results are given in Section $\mathrm{V}$ for some cases. It is noteworthy to point out that these two probabilities are usually not the same each other and are also not the same as the steady-state probability when there are $n$ calls in the cell. Some relations among these three steady-state probabilities are included in Section V.

Now, we study the distribution of the ACCT. At first, it is readily shown that

$$
P(X<x, A)=p_{0} u(x)
$$

where $u(x)$ is an unit step function, i.e., $u(t)$ is one if $x>0$ and zero otherwise.

Let $H$ denote the generic form of the requested call connection time and $R_{i}$ denote the cell residence time when a mobile

$$
b_{i}= \begin{cases}i \mu_{0}, & \text { if } 0 \leq i \leq K \\ K \mu_{0}+(i-K)\left(\mu_{1}-\mu_{0}\right), & \text { if } K+1 \leq i \leq K+C \\ K \mu_{0}+C\left(\mu_{1}-\mu_{0}\right)+(i-K-C) \mu_{2}, & \text { if } K+C+1 \leq i \leq K+C+N\end{cases}
$$


traverses the $i$ th cell during its call connection. Due to the i.i.d. assumption, $R_{i}(i=1,2, \ldots)$ are i.i.d. random variables. Let $R$ denote its generic form. By a direct probability argument, we have

$$
\begin{aligned}
& P(X<x, \bar{A} C) \\
&=\left(1-p_{0}\right) \sum_{n=0}^{\infty}\left(1-p_{f}\right)^{n} \\
& \times P\left(H+\sum_{k=0}^{n} W_{k}<x \sum_{k=0}^{n} R_{k} \leq H<\sum_{k=0}^{n+1} R_{k}\right) \\
& P\left(X<x, \bar{A} B_{1}\right)=\left(1-p_{0}\right) \sum_{n=0}^{\infty}\left(1-p_{f}\right)^{n} p_{f} \\
& \quad P\left(\sum_{k=0}^{n+1} R_{k}<H \leq \sum_{k=0}^{n}\left(R_{k}+W_{k}\right)\right. \\
&\left.\quad+R_{n+1}+\tau_{n+1}<x\right)
\end{aligned}
$$

and

$$
\begin{aligned}
P\left(X<x, \bar{A} B_{2}\right)= & \left(1-p_{0}\right) \sum_{n=0}^{\infty}\left(1-p_{f}\right)^{n} p_{f} \\
& \times P\left(\sum_{k=0}^{n}\left(R_{k}+W_{k}\right)+R_{n+1}+\tau_{n+1}<x\right. \\
& \left.\sum_{k=0}^{n}\left(R_{k}+W_{k}\right)+R_{n+1}+\tau_{n+1}<H\right)
\end{aligned}
$$

where $W_{0}=R_{0}=0, W_{i}(i=1,2, \ldots$,$) is the actual waiting$ time of a handoff call in the $i$ th handoff area during its connection period, and $\tau_{n}$ is the handoff call's maximum queueing time in the $n$th handoff area.

Based on the results in (3.3), (3.4), (3.5), and (3.6), we obtain the distribution function for the ACCT as shown in (3.7) at the bottom of the page.

We are more interested in the closed expressions of the L-S transform and also the expectation of the actual call connection time in the research. For this purpose, we first need to know some properties of distribution of random waiting time
$W_{i}$ based on the result in (3.7). We then find the simple closed expressions for both of these two interested measures.

Based on the assumptions of the network, it is easily to know that $W_{1}, W_{2}, \ldots$ are i.i.d. random variables. Let $W$ be its generic form. By a direct probability argument, we have

$$
\begin{aligned}
P(W \geq x)= & \sum_{k=0}^{L} \bar{\pi}_{k} P(W \geq x \mid \text { arriving handoff call finds } \\
& k \text { calls in the handoff area }) \\
= & \left(\sum_{i=0}^{K+C-1} \bar{\pi}_{i}+\bar{\pi}_{K+C+N}\right)[1-u(x)] \\
& +\sum_{j=0}^{N-1} \bar{\pi}_{K+C+j} P\left(\phi_{0}+\phi_{1}+\cdots+\phi_{j} \geq x\right) \\
= & \left(\sum_{i=0}^{K+C-1} \bar{\pi}_{i}+\bar{\pi}_{K+C+N}\right)[1-u(x)] \\
& +\sum_{j=0}^{N-1} \bar{\pi}_{K+C+j} \\
& \times \sum_{i=0}^{j}\left(\prod_{l=0, l \neq i}^{j} \frac{b_{K+C+l}}{b_{K+C+l}-b_{K+C+i}}\right) e^{-b_{K+C+i} x} .
\end{aligned}
$$

Therefore, the Laplace-Stieltjes transform of the probability distribution of random variable $W$ is given by

$$
\begin{aligned}
w^{*}(s)= & \int_{0}^{+\infty} e^{-s x} d P(W<x) \\
= & \sum_{i=0}^{K+C-1} \bar{\pi}_{i} \\
& +\sum_{j=0}^{N-1} \bar{\pi}_{K+C+j}\left[\prod_{i=0}^{j}\left(\frac{b_{K+C+j}}{s+b_{K+C+j}}\right)\right]+\bar{\pi}_{K+C+N}
\end{aligned}
$$

and the average actual waiting time of a handoff call in the handoff area is given by

$$
E[W]=\sum_{j=0}^{N-1} \sum_{i=0}^{j} \frac{\bar{\pi}_{K+C+j}}{b_{K+C+i}} .
$$

$$
\begin{aligned}
P(X<x)= & p_{0} u(x)+\left(1-p_{0}\right) \sum_{n=0}^{\infty}\left(1-p_{f}\right)^{n}\left[P\left(H+\sum_{k=0}^{n} W_{k} \leq x ; \sum_{k=0}^{n} R_{k}<H<\sum_{k=0}^{n+1} R_{k}\right)\right. \\
& \left.+p_{f} P\left(\sum_{k=0}^{n}\left(R_{k}+W_{k}\right)+R_{n+1}+\tau_{n+1}<x, \sum_{k=0}^{n+1} R_{k}<H\right)\right] \\
= & p_{0} u(x)+\left(1-p_{0}\right) \sum_{n=0}^{\infty}\left(1-p_{f}\right)^{n} \\
& \times \int_{0}^{x}\left[h e^{-(h+r) t} \frac{(r t)^{n}}{n !} P\left(\sum_{k=0}^{n} W_{k}<x-t\right)+p_{f} e^{-(h+r) t} \frac{r(r t)^{n}}{n !} P\left(\sum_{k=0}^{n} W_{k}+\tau_{n+1}<x-t\right)\right] d t .
\end{aligned}
$$


Now, from results and (3.7), if we denote by $a^{*}(s)$ the L-S transform of probability distribution of the ACCT $X$, we have

$$
\begin{aligned}
a^{*}(s)= & p_{0}+\left(1-p_{0}\right) \sum_{n=0}^{\infty}\left(1-p_{f}\right)^{n} \\
& \times\left[\frac{h}{r}\left(w^{*}(s)\right)^{n}\left(\frac{r}{r+h+s}\right)^{n+1}\right. \\
& \left.+p_{f} \frac{d+a}{(d+a+s)}\left(w^{*}(s)\right)^{n}\left(\frac{r}{r+h+s}\right)^{n+1}\right] \\
= & p_{0}+\frac{\left(1-p_{0}\right)}{h+r+s-r\left(1-p_{f}\right) w^{*}(s)} \\
& \times\left[h+r p_{f} \frac{(d+a)}{d+a+s}\right] .
\end{aligned}
$$

From this result, it is readily to know that the expected ACCT $E[X]$ is then given by

$$
\begin{aligned}
E[X]=\lim _{s \rightarrow 0}\left(\frac{1-a^{*}(s)}{s}\right)=\frac{1-p_{0}}{h+r p_{f}} \\
\times\left[1+r\left(1-p_{f}\right) E[W]+\frac{r p_{f}}{d+a}\right] .
\end{aligned}
$$

Remark 4: From the above simple formulae in (3.11) and (3.12), we can easily find the related results for the four channel allocation schemes described in the introduction. For example, if let $N=0$ in the equation (3.12), we have $E X=\left(1-p_{0} /\left(h+r p_{f}\right)\right)$. This is the same as that obtained for RCS in [18] when $h_{1}=h_{2}=h$, by noticing that in this case $d$ could be reasonably deemed as $\infty$. In fact, as for NPS and SRS, the formulae of the average ACCT are the same as that in RCS with a slightly modification of the expressions for $p_{0}$ and $p_{f}$ only based on different schemes. The formula of the average ACCT for the case of QPS, however, can not be so simple as those in RCS, NPS and SRS. This formula can directly be obtained from result in (3.12) by applying $C=0$.

\section{Complete Calls and Incomplete Calls}

As we mentioned earlier, it is important to distinguish complete calls from incomplete calls. Due to such consideration, we are interested in the call completion probability, the call dropping probability, and the actual call connections times for complete calls and incomplete calls.

\section{A. Probabilities}

Based on the relationship between the actual call connection time and the requested call connection time of a new call, we can classify the actual call connection times into three categories.

- The first category occurs when the new call has failed to be connected. This means that the actual call connection time is zero.

- The second category occurs when the new call is connected and completed. This implies that:

- the call completes all necessary handoffs successfully;

- the ACCT is greater than zero and is also greater than or equal to the RCCT.
- The final category occurs when the new call is connected but is eventually forced to terminate. This implies that the ACCT is greater than zero, and that:

- the ACCT is greater than or equal to the RCCT, but the call is eventually forced to terminate during a handoff, or

- the ACCT is less than the RCCT.

Based on above observation and the results in equations (3.4), (3.5), and (3.6), we have the following results.

- The probability that a call is complete (the call completion probability) is

$$
\begin{aligned}
p_{C} & =P(\bar{A} C)=\lim _{x \rightarrow \infty} P(X<x, \bar{A} C) \\
& =\left(1-p_{0}\right) \sum_{n=0}^{\infty}\left(1-p_{f}\right)^{n} P\left(\sum_{k=0}^{n} R_{k}<H<\sum_{k=0}^{n+1} R_{k}\right) \\
& =\frac{\left(1-p_{0}\right) h}{h+r p_{f}}
\end{aligned}
$$

- For incomplete calls, we have the following two situations.

1) The probability that a call is incomplete and the actual call connection time is greater than or equal to the requested call holding time is given by

$$
\begin{aligned}
p_{I 1}= & P\left(\bar{A} B_{1}\right)=\lim _{x \rightarrow \infty} P\left(X<x, \bar{A} B_{1}\right) \\
= & \left(1-p_{0}\right) p_{f} \sum_{n=0}^{\infty}\left(1-p_{f}\right)^{n} \\
& \times P\left(\sum_{k=0}^{n+1} R_{k}<H \leq \sum_{k=0}^{n}\left(R_{k}+W_{k}\right)+R_{n+1}+\tau_{n+1}\right) \\
= & \left(1-p_{0}\right) p_{f} \sum_{n=0}^{\infty}\left(1-p_{f}\right)^{n}\left(\frac{r}{h+r}\right)^{n+1} \\
& \times\left[1-\frac{(d+a)\left[w^{*}(h)\right]^{n}}{d+a+h}\right] \\
= & \left(1-p_{0}\right) r p_{f}\left[\frac{1}{h+r p_{f}}\right. \\
& \left.-\frac{(d+a)}{(d+a+h)\left[(h+r)-r\left(1-p_{f}\right) w^{*}(h)\right]}\right]
\end{aligned}
$$

2) The probability that a call is incomplete and the actual call connection time is less than the requested call holding time is given by

$$
\begin{aligned}
p_{I 2}= & P\left(\bar{A} B_{2}\right)=\lim _{x \rightarrow \infty} P\left(X<x, \bar{A} B_{2}\right) \\
= & \left(1-p_{0}\right) p_{f} \sum_{n=0}^{\infty}\left(1-p_{f}\right)^{n} \\
& \times P\left(\sum_{k=0}^{n}\left(R_{k}+W_{k}\right)+R_{n+1}+\tau_{n+1}<H\right) \\
= & \left(1-p_{0}\right) p_{f} \sum_{n=0}^{\infty}\left(1-p_{f}\right)^{n}\left(\frac{r}{h+r}\right)^{n+1} \\
& \times\left[\frac{(d+a)\left[w^{*}(h)\right]^{n}}{d+a+h}\right] \\
= & \frac{\left(1-p_{0}\right) r p_{f}(d+a)}{(d+a+h)\left[h+r-r\left(1-p_{f}\right) w^{*}(h)\right]} .
\end{aligned}
$$


Therefore, the probability that a call is incomplete is

$$
p_{I}=p_{I 1}+p_{I 2}=\frac{\left(1-p_{0}\right) r p_{f}}{h+r p_{f}} .
$$

It is easy to see that $p_{0}+p_{I}+p_{C}=1$. This is consistent with the intuition that a new call may be blocked in the initial cell, or may be connected with the initial cell and ends with an incomplete call or a complete call. It is also noted that our results are the same as those in [20] for a specific scheme.

\section{B. Average ACCTs for Complete Calls and Incomplete Calls}

The average actual call connection times for complete calls and incomplete calls are of great interest. We determine analytical results for them in this section.

Let $I_{D}$ denote the indicator function of the event $D$, that is, $I_{D}$ is equal to one if $D$ occurs and zero otherwise. Let $X I_{C}$ and $X I_{B}$ denote the actual call connection times for a complete call and an incomplete call, respectively. From (3.4), the average ACCT for a complete call is given by

$$
\begin{aligned}
E\left[X I_{C}\right]= & \left(1-p_{0}\right) \sum_{n=0}^{\infty}\left(1-p_{f}\right)^{n} \\
& \times \int_{0}^{\infty}\left[(t+n E[W]) h e^{-(h+r) t} \frac{(r t)^{n}}{n !}\right] d t \\
= & \frac{\left(1-p_{0}\right) h}{\left(h+r p_{f}\right)^{2}}\left[1+r\left(1-p_{f}\right)\right] .
\end{aligned}
$$

From (3.5) and (3.6), we obtain the average ACCT for an incomplete call

$$
\begin{aligned}
E\left[X I_{B}\right]= & \left(1-p_{0}\right) p_{f} \sum_{n=0}^{\infty}\left(1-p_{f}\right)^{n} \\
& \times \int_{0}^{\infty} \frac{r(r t)^{n} e^{-(h+r) t}}{n !}\left[t+n E[W]+\frac{1}{d+r}\right] d t \\
= & \left(1-p_{0}\right) p_{f}\left[\frac{r^{2}\left(1-p_{f}\right) E[W]}{\left(h+r p_{f}\right)^{2}}\right. \\
& \left.+\frac{r}{(d+a)\left(h+r p_{f}\right)}+\frac{r}{\left(h+r p_{f}\right)^{2}}\right]
\end{aligned}
$$

Notice that the result of average ACCT in (3.12) can also be obtained by substituting (4.17) and (4.18) into the expression $E[X]=E X I_{B}+E X I_{C}$.

\section{Computation of Probabilities $p_{0}$ AND $p_{f}$}

We observe that in order to apply our results, we need to find the new call blocking probability $p_{0}$ and the forced termination probability of a handoff call $p_{f}$. According to the results in Section III, we know that these two probabilities are closely dependent on the steady state probabilities of number of the calls in the cell when a handoff call and an new call arrive to the cell, respectively. Now, let $\pi_{n}$ denote the steady state probability when there are $n$ calls in the cell. It is straightforward to observe that in general $\bar{\pi}_{n} \neq \pi_{n}$ if the handoff arrival process is not Poisson process and that in general $\tilde{\pi}_{n} \neq \pi_{n}$ if the new arrival process is not Poisson process. To show this difference and to finally find the probabilities $p_{0}$ and $p_{f}$, in this section, we present the results for cases when the call arrival processes are modeled by the independent call Poisson arrival processes, the dependent call arrival processes and the correlated call arrival processes, respectively.

\section{A. Independent Call Arrival Processes}

In this case, the new calls and handoff calls are assumed as Poisson arrival processes and are independent of each other. According to the well-known Poisson arrivals see time averages (PASTA) result in queueing theory, we know that in this case $\bar{\pi}_{n}=\tilde{\pi}_{n}=\pi_{n}$ for all possible $n$. Now, suppose that the new call arrival process is Poisson with rate $\lambda$, and that the handoff call arrival process is Poisson with rate $\eta$. By a direct probabilistic analysis (Markov chain theory), we have the equation at the bottom of the page, where $L=K+C+N$ and the first equation at the bottom of the next page.

Therefore, from the results in (3.2) and (3.1), the new call blocking probability is given by

$$
p_{0}=\sum_{n=K}^{K+C+N} \pi_{n} .
$$

and the probability that a handoff call is forced to terminate in a handoff area is given by

$$
p_{f}=\pi_{K+C+N}+\sum_{j=0}^{N-1} \pi_{K+C+j} \frac{(j+1) \mu_{2}}{b_{K+C+j+1}} .
$$

\section{B. Dependent Call Arrival Processes}

In some wireless networks, call arrival processes may be dependent on the number of call connections in a cell [4], [19]. In this case, $\bar{\pi}_{n}$ and $\tilde{\pi}_{n}$ will be different from $\pi_{n}$. Suppose that

$$
\bar{\pi}_{n}= \begin{cases}\frac{(\eta+\lambda)^{n} \bar{\pi}_{0}}{n ! \mu_{0}^{n}}, & \text { if } 0 \leq n \leq K \\ \frac{(\eta+\lambda)^{K} \eta^{n-K} \bar{\pi}_{0}}{K ! \mu_{0}^{K} \prod_{j=1}^{n-K}\left[K \mu_{0}+j\left(\mu_{1}-\mu_{0}\right)\right]}, & \text { if } K+1 \leq n \leq K+C \\ \frac{(\eta+\lambda)^{K} \eta^{n-K} \bar{\pi}_{0}}{n-K-C}, & \text { if } K+C+1 \leq n \leq L . \\ K ! \mu_{0}^{K} \prod_{j=1}^{C}\left[K \mu_{0}+j\left(\mu_{1}-\mu_{0}\right)\right] \prod_{s=1}^{n-K}\left[K \mu_{0}+C\left(\mu_{1}-\mu_{0}\right)+s \mu_{2}\right] & \end{cases}
$$


the new call arrival process is Poisson with rate $\lambda_{n}$ and that the handoff call arrival process is Poisson with rate $\eta_{n}$ when there are $n$ calls in the cell. Using a similar argument as that in [19], we obtain the second equation at the bottom of the page, where

$$
\begin{aligned}
\pi_{0}= & +\sum_{n=0}^{K} \prod_{i=1}^{n}\left(\frac{\lambda_{i-1}+\eta_{i-1}}{i \mu_{0}}\right) \\
& +\sum_{n=K+1}^{K+C} \prod_{i=1}^{K}\left(\frac{\lambda_{i-1}+\eta_{i-1}}{i \mu_{0}}\right) \\
& \times \prod_{j=1}^{n-K}\left(\frac{\eta_{K+j-1}}{K \mu_{0}+j\left(\mu_{1}-\mu_{0}\right)}\right) \\
& +\sum_{n=K+C+1}^{K+C+N} \prod_{i=1}^{K}\left(\frac{\lambda_{i-1}+\eta_{i-1}}{i \mu_{0}}\right) \\
& \times \prod_{j=1}^{C}\left(\frac{\eta_{K+j-1}}{K \mu_{0}+j\left(\mu_{1}-\mu_{0}\right)}\right) \\
& \times \prod_{s=1}^{n-K}\left(\frac{\eta_{K+C+s-1}}{K \mu_{0}+C\left(\mu_{1}-\mu_{0}\right)+s \mu_{2}}\right) .
\end{aligned}
$$

Now, we consider the average number of handoff calls over a longer period of time, say $\theta$, when the system is in equilibrium. We observe that the average number of handoff call arrivals is $\sum_{j=0}^{K+N+C} \eta_{j} \pi_{j} \theta$ and that the average number of handoff calls finding $n$ calls in the current cell is $\eta_{n} \pi_{n} \theta$. Thus, the probability that an arriving handoff call finds that there are $n$ calls in the cell is

$$
\bar{\pi}_{n}=\frac{\eta_{n} \pi_{n}}{\sum_{j=0}^{K+N+C} \eta_{j} \pi_{j}}
$$

Similarly, the probability that a new call finds that there are $n$ calls in the cell is

$$
\tilde{\pi}_{n}=\frac{\lambda_{n} \pi_{n}}{\sum_{j=0}^{K+N+C} \lambda_{j} \pi_{j}}
$$

Therefore, from the results in (3.1) and (3.2), we obtain the new call blocking probability is

$$
p_{0}=\frac{\sum_{j=K}^{K+N+C} \lambda_{j} \pi_{j}}{\sum_{j=0}^{K+N+C} \lambda_{j} \pi_{j}}
$$

and the probability that a handoff call is forced to terminate in a handoff area is

$p_{f}=$

$$
\frac{\left(\eta_{K+C+N} \pi_{K+C+N}+\sum_{j=0}^{N-1} \eta_{K+C+j} \pi_{\left.K+C+j \frac{(j+1) \mu_{2}}{b_{K+C+j+1}}\right)}^{K+N+C} \sum_{j=0}^{K+N} \eta_{j}\right.}{.}
$$

\section{Correlated Call Arrival Processes}

In this situation, we consider the MAP, which contains both phase type renewal processes and correlated arrival streams, such as the Markov modulated Poisson process (MMPP), as special cases. For the application of the MAP in the PCS networks, please refer to [17] and the references therein. More precisely, let $D$ be the irreducible infinitesimal generator of an $m$ state Markov process. The sojourn time in state $i$ is exponentially distributed with parameter $\lambda_{i}, i=1,2, \ldots, m$. At the end of the sojourn time in state $i$, three kinds of transitions may take place:

$$
\begin{aligned}
\bar{\pi}_{0}= & \sum_{n=0}^{K} \frac{(\eta+\lambda)^{n}}{n ! \mu_{0}^{n}} \\
& +\sum_{n=K+1}^{K+C} \frac{(\eta+\lambda)^{K} \eta^{n-K}}{K ! \mu_{0}^{K} \prod_{j=1}^{n-K}\left[K \mu_{0}+j\left(\mu_{1}-\mu_{0}\right)\right]} \\
& +\sum_{n=K+C+1}^{K+C+N} \frac{(\eta+\lambda)^{K} \eta^{n-K}}{K ! \mu_{0}^{K} \prod_{j=1}^{C}\left[K \mu_{0}+j\left(\mu_{1}-\mu_{0}\right)\right] \prod_{s=1}^{n-K-C}\left[K \mu_{0}+C\left(\mu_{1}-\mu_{0}\right)+s \mu_{2}\right]}
\end{aligned}
$$

$$
\pi_{n}= \begin{cases}\pi_{0} \prod_{i=1}^{n}\left(\frac{\lambda_{i-1}+\eta_{i-1}}{i \mu_{0}}\right), & \text { if } 1 \leq n \leq K \\ \pi_{0} \prod_{i=1}^{K}\left(\frac{\lambda_{i-1}+\eta_{i-1}}{i \mu_{0}}\right) \prod_{j=1}^{n-K}\left(\frac{\eta_{K+j-1}}{K \mu_{0}+j\left(\mu_{1}-\mu_{0}\right)}\right), & \text { if } K+1 \leq n \leq K+C \\ \pi_{0} \prod_{i=1}^{K}\left(\frac{\lambda_{i-1}+\eta_{i-1}}{i \mu_{0}}\right) \prod_{j=1}^{C}\left(\frac{\eta_{K+j-1}}{K \mu_{0}+j\left(\mu_{1}-\mu_{0}\right)}\right) \prod_{s=1}^{n-K-C}\left(\frac{\eta_{K+C+s-1}}{K \mu_{0}+C\left(\mu_{1}-\mu_{0}\right)+s \mu_{2}}\right), & \text { if } K+C+1 \leq n \leq L\end{cases}
$$


1) with probability $p_{0}(i, k),(k=1,2, \ldots, m, k \neq i)$, a transition occurs to the state $k$, without any kinds of call arrivals;

2) with probability $p_{N}(i, k),(k=1,2, \ldots, m)$, a transition occurs to the state $k$, with a new call arrival;

3) with probability $p_{H}(i, k),(k=1,2, \ldots, m)$, a transition occurs to the state $k$, with a handoff call arrival.

Obviously, for each fixed $i$, the sum of all probabilities above, over all possible value of $k$ equals one, i.e.,

$$
\sum_{k \neq i}^{m} p_{0}(i, k)+\sum_{k=1}^{m} p_{N}(i, k)+\sum_{k=1}^{m} p_{H}(i, k)=1 .
$$

With this notation, if we denote the matrices $D_{0}, D_{N}$, and $D_{H}$ by $\left(D_{0}\right)_{i, i}=-\lambda_{i},\left(D_{0}\right)_{i, k}=\lambda_{i} p_{0}(i, k),\left(D_{N}\right)_{i, j}=\lambda_{i} p_{N}(i, j)$ and $\left(D_{H}\right)_{i, j}=\lambda_{i} p_{H}(i, j)$ for $i \neq k$ and $i, j, k=1,2, \ldots, m$, then the MAP is a semi-Markovian arrival process with transition matrix

$$
\begin{aligned}
Q(t) & =\int_{0}^{t} e^{D_{0} x} d x\left(D_{H}+D_{N}\right) \\
& =\left(I-e^{D_{0} t}\right)\left(-D_{0}^{-1}\right)\left(D_{N}+D_{H}\right) .
\end{aligned}
$$

Let $\boldsymbol{\alpha}$ be the stationary probability vector of the generator $D$, i.e., $\boldsymbol{\alpha}$ satisfies $\boldsymbol{\alpha} D=0$ and $\boldsymbol{\alpha e}=1$ where $\mathbf{e}$ is a column vector of 1's. We obtain that $D=D_{0}+D_{N}+D_{H}$ and that the new call arrival rate, say $\lambda_{N}$, of the MAP is $\lambda_{N}=\boldsymbol{\alpha} D_{N} \mathbf{e}$ and that the handoff call arrival rate, say $\lambda_{H}$, of the MAP is $\lambda_{H}=\boldsymbol{\alpha} D_{H} \mathbf{e}$.

Denote by $\pi_{n, i}(n=0,1, \ldots, L ; i=1,2, \ldots, m)$ the steady-state probability that there are $n$ calls in the cell and that the arrival process is in the $i$ th phase. Let $\pi_{n}=\left(\pi_{n, 1}, \pi_{n, 2}, \ldots, \pi_{n, m}\right)$. From [11, Lemma 3], if we let $\prod_{i=1}^{n} \beta_{i}=\beta_{1} \beta_{2} \ldots \beta_{n}$ for matrices $\beta_{1}, \ldots, \beta_{n}$, we find [17]:

$$
\pi_{n}=\pi_{0} \prod_{i=1}^{n}\left[A_{i-1}\left(-C_{i}\right)^{-1}\right] \quad n=1,2, \ldots, L
$$

where

$$
\begin{aligned}
A_{i}= \begin{cases}D_{N}+D_{H}, & \text { if } 0 \leq i \leq K-1 \\
D_{H}, & \text { if } K \leq i \leq L-1\end{cases} \\
E_{i}= \begin{cases}D_{0}, & \text { if } i=0 \\
D_{0}-b_{i} I, & \text { if } 1 \leq i \leq K-1 \\
\left(D_{0}+D_{N}\right)-b_{i} I, & \text { if } K \leq i \leq K+C+N-1 \\
-b_{K+C+N} I, & \text { if } i=K+C+N .\end{cases}
\end{aligned}
$$

$I$ is an unit matrix, and $\pi_{0}$ satisfies $\pi_{0} C_{0}=0$ and

$$
\boldsymbol{\pi}_{0}\left[I+\sum_{n=1}^{L} \prod_{i=1}^{n}\left[A_{i-1}\left(-C_{i}\right)^{-1}\right]\right] \mathbf{e}=1 .
$$

The $C_{i}(i=0,1, \ldots, L)$ are recursively determined by $C_{L}=$ $E_{L}$ and

$$
C_{n}=E_{n}+A_{n}\left(-C_{n+1}^{-1}\right) b_{n+1} \quad(n=0,1, \ldots, L-1) .
$$

Since the total handoff arrival rate is $\alpha D_{N} \mathbf{e}$ and the new call arrival rate is $\boldsymbol{\alpha} D_{H} \mathbf{e}$, we know that the probability that an arriving new call finds $n$ calls in the cell is

$$
\tilde{\pi}_{n}=\frac{\boldsymbol{\pi}_{n} D_{N} \mathbf{e}}{\boldsymbol{\alpha} D_{N} \mathbf{e}} \quad n=0,1, \ldots, L
$$

and an arriving handoff call finds $n$ calls in the cell is

$$
\bar{\pi}_{n}=\frac{\boldsymbol{\pi}_{n} D_{H} \mathbf{e}}{\boldsymbol{\alpha} D_{H} \mathbf{e}} \quad n=0,1, \ldots, L .
$$

Therefore, from the results in (3.1) and (3.2), we obtain the new call blocking probability is

$$
p_{0}=\frac{\left(\sum_{n=K}^{K+N+C} \pi_{n}\right) D_{N} \mathbf{e}}{\boldsymbol{\alpha} D_{N} \mathbf{e}}
$$

and the probability that a handoff call is forced to terminate in a handoff area is

$$
p_{f}=\frac{\left(\boldsymbol{\pi}_{K+C+N} D_{H} \mathbf{e}+\sum_{j=0}^{N-1} \boldsymbol{\pi}_{K+C+j} D_{H} \mathbf{e}_{b_{K+C+j+1}}\right)}{\boldsymbol{\alpha} D_{H} \mathbf{e}} .
$$

\section{CONCLUSION}

In this paper, we analytically characterize the actual call connection time and related performance metrics for wireless mobile networks under a newly proposed general channel allocation scheme. Explicit formulae for the distribution and the expectation of the ACCT are obtained. The call completion probability, the call drop probability and the average actual call connection times for both complete calls and incomplete calls are also derived. With the analytical results for actual call connection times, billing rate planning schemes for wireless network services can be evaluated and designed. More importantly, the analytical techniques developed in this paper may be useful for performance analysis of other telecommunications systems.

\section{ACKNOWLEDGMENT}

The authors would like to express their sincere appreciation to the editor and the reviewers for their constructive comments and suggestions, which significantly improved the quality of this paper.

\section{REFERENCES}

[1] F. Baccelli, P. Boyer, and G. Hebuterne, "Single server queues with impatient customers," Adv. Appl. Probability, vol. 16, pp. 887-905, 1984.

[2] D. Y. Barrer, "Queueing with impatient customers and indifferent clerks," Oper. Res., vol. 5, pp. 644-649, 1957.

[3] —, "Queueing with impatient customers and ordered service," Oper. Res., vol. 5, pp. 650-656, 1957.

[4] A. Brandt and M. Brandt, "On the $M(n) / M(n) / s$ queue with impatient calls," Perform. Eval., vol. 35, no. 1-2, pp. 1-18, 1999.

[5] Y. Fang, "Hyper-Erlang distribution models and its application in wireless mobile networks," Wireless Networks (WINET), vol. 7, pp. 211-219, 2001.

[6] Y. Fang and I. Chlamtac, "Teletraffic analysis and mobility modeling of PCS networks," IEEE Trans. Commun., vol. 47, pp. 1062-1072, July 1999.

[7] Y. Fang, I. Chlamtac, and Y. B. Lin, "Channel occupancy times and handoff rate for mobile computing and PCS networks," IEEE Trans. Comput., vol. 47, pp. 679-692, June 1998.

[8] — "Modeling PCS networks under general call holding times and cell residence time distributions," IEEE/ACM Trans. Networking, vol. 5, pp. 893-906, Dec. 1997.

[9] - "Call performance for a PCS network," IEEE J. Select. Areas Commun., vol. 15, pp. 1568-1581, Oct. 1997.

[10] Y. Fang and Y.Zhang, "Call admission control schemes and performance analysis in wireless mobile networks," IEEE Trans. Veh. Technol., vol. 51, pp. 371-382, Mar. 2002. 
[11] D. P. Gaver, P. A. Jacobs, and G. Latouche, "Finite birth-and-death models in randomly changing environments," Adv. Appl. Probability, vol. 16, pp. 715-731, 1984.

[12] R. B. Haugen and E. Skogan, "Queueing systems with stochastic time out," IEEE Trans. Commun., vol. COM-28, pp. 1984-1989, 1980.

[13] D. Hong and S. S. Rappaport, "Traffic model and performance analysis for cellular mobile radio telephone systems with prioritized and nonprioritized handoff procedures," IEEE Trans. Veh. Technol., vol. VT-35, pp. 77-92, 1986.

[14] M. Ivanovich, M. Zukerman, P. Fitzpatrick, and M. Githts, "Performance between circuit allocation schemes for half- and full-rate connections in GSM,” IEEE Trans. Veh. Technol., vol. 47, pp. 790-797, Aug. 1998.

[15] M. Kawarazaki, H. Saito, and H. Yamada, "An analysis of statistica multiplexing in an ATM transport network," IEEE J. Select. Areas Commun., vol. 9, pp. 359-367, Apr. 1991.

[16] M. D. Kulavaratharasah and A. H. Aghvami, "Teletraffic performance evaluation of microcellular personal communication networks (PCNs) with prioritized handoff procedures," IEEE Trans. Veh. Technol., vol. 48, pp. 137-152, Jan. 1999

[17] W. Li and A. S. Alfa, "A PCS network with correlated arrival process and splitted-rate channels," IEEE J. Select. Areas Commun., vol. 17, pp. 1318-1325, July 1999.

[18] _ "Channel reservation for hand-off calls in a PCS network," IEEE Trans. Veh. Technol., vol. 49, pp. 95-104, Jan. 2000.

[19] W. Li, K. Makki, and N. Pissinou, "Performance analysis of a PCS network with state-dependent calls arrival processes and impatient calls," Comput. Commun., vol. 25, pp. 507-515, 2002

[20] Y. B. Lin, S. Mohan, and A. Noerpel, "Queueing priority channel assignment strategies for PCS handoff and initial access," IEEE Trans. Veh. Technol., vol. 43, pp. 704-712, Aug. 1994

[21] Y. B. Lin, R. Anthony, and D. J. Harasty, "The subrate channel assignment strategy for PCS hand-offs," IEEE Trans. Veh. Technol., vol. 45, pp. 122-130, Feb. 1996.

[22] K. Sriram and W. Whitt, "Characterizing superposition arrival processes in an ATM transport network," IEEE J. Select. Areas Commun., vol. 4, pp. 359-367, 1986

[23] P. V. Orlik and S. S. Rappaport, "A model for teletraffic performance and channel holding time characterization in wireless cellular communication with general session and dwell time distributions," IEEE J. Select. Areas Commun., vol. 16, pp. 788-803, June 1998.

[24] V. Paxon and S. Floyd, "Wide area traffic: The failure of poisson modeling," IEEE/ACM Trans. Networking, vol. 3, pp. 226-244, June 1995.

[25] S. S. Rappaport, "The multiple-call hand-off problem in high-capacity cellular communications systems," IEEE Trans. Veh. Technol., vol. 40, pp. 546-557, 1991.

[26] S. Tekinary and B. Jabbari, "Handover and channel assignment in mobile cellular networks," IEEE Commun. Mag., pp. 42-46, Nov. 1991.

[27] Y. Q. Zhao and S. A. Alfa, "Performance analysis of a telephone system with both patient and impatient customers," Telecommun. Syst., vol. 4 , pp. 201-215, 1995

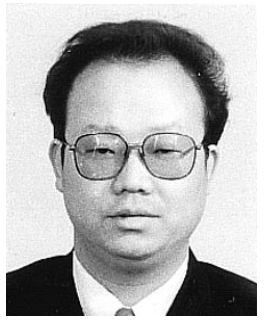

Wei Li (M'99) received the B.Sc. degree from Shannxi Normal University, Xian, China, in 1982 , the M.Sc. degree from Hebei University of Technology, Tianjin, China, in 1987, and the Ph.D. degree from the Chinese Academy of Sciences, Beijing, China, in 1994

$\mathrm{He}$ is an Associate Professor in the Department of Electrical Engineering and Computer Science, University of Toledo, Toledo, OH. From 2000 to 2002, he was named as the Bell South/BORSF Professor in Telecommunications. From 1999 to 2002, he was an Assistant Professor in the Department of Electrical and Computer Engineering, University of Louisiana, Lafayette. He engaged in research and teaching at the University of Manitoba, Winnipeg, Canada, and the University of Winnipeg, Winnipeg, Canada, from 1995 to 1999 . He was once a Senior Researcher at the University of Newcastle, England and an Associate Researcher at the Chinese Academy of Sciences. His current research interests are in the evaluation, design and implementation of teletraffic models for personal communications services networks, wireless networks, and mobile satellite communications networks as well as the applications in these areas of operations research, queueing networks, and reliability networks.

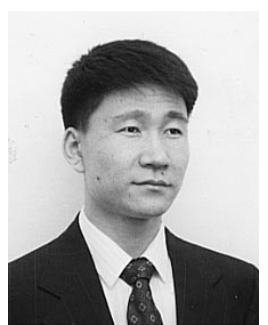

Yuguang Fang (S'92-M'94-SM'99) received the B.S. and M.S. degrees in mathematics from Qufu Normal University, Qufu, Shandong, China, in 1984 and 1987, respectively, the Ph.D degree in systems and control engineering from the Department of Systems, Control and Industrial Engineering, Case Western Reserve University, Cleveland, $\mathrm{OH}$, in January 1994, and the Ph.D degree in electrical engineering from the Department of Electrical and Computer Engineering, Boston University, Boston, MA, in May 1997.

From 1987 to 1988, he held research and teaching positions in both the Department of Mathematics and the Institute of Automation at Qufu Normal University. From September 1989 to December 1993, he was a Teaching/Research Assistant in the Department of Systems, Control and Industrial Engineering, Case Western Reserve University, where he held a Research Associate position from January 1994 to May 1994. From June 1994 to August 1995, he held a Postdoctoral position in the Department of Electrical and Computer Engineering, Boston University. From September 1995 to May 1997, he was a Research Assistant in the Department of Electrical and Computer Engineering, Boston University. From June 1997 to July 1998, he was a Visiting Assistant Professor in the Department of Electrical Engineering, University of Texas, Dallas. From July 1998 to May 2000, he was an Assistant Professor in the Department of Electrical and Computer Engineering, New Jersey Institute of Technology, Newark. Since May 2000, he has been an Assistant Professor in the Department of Electrical and Computer Engineering, University of Florida, Gainesville, FL. His research interests span many areas including wireless networks, mobile computing, mobile communications, automatic control, and neural networks. He has published over 80 papers in refereed professional journals and conferences. He is an Editor for ACM Wireless Networks, an Area Editor for ACM Mobile Computing and Communications Review, an Associate Editor for Wiley International Journal on Wireless Communications and Mobile Computing. He is also actively involved with many professional conferences such as ACM MobiCom'01, IEEE INFOCOM'98, INFOCOM'00, IEEE WCNC'02, WCNC'00 (Technical Program Vice-Chair), WCNC'99, and the International Conference on Computer Communications and Networking (IC3N'98) (Technical Program Vice-Chair).

Dr. Fang has also engaged in many professional activities. He is a Member of ACM. He is an Editor for IEEE TRANSACTIONS ON COMMUNICATIONS, an Editor for IEEE JOURNAL ON WIRELESS COMMUNICATIONS. He is Feature Edito for Scanning the Literature in IEEE PERSONAL COMMUNICATIONS. He has received the prestigious National Science Foundation Faculty Early Career Award in 2001 and the Office of Naval Research Young Investigator Award in 2002. He is listed in Marquis Who's Who in Science and Engineering, Who's Who in America, and Who's Who in World.

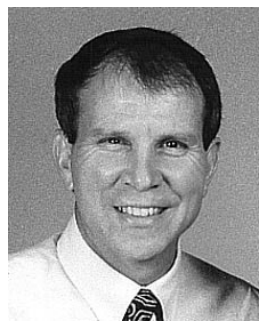

Robert R. Henry (M'67-SM'83) received the B.S.E.E. and M.S.E.E. degrees from the University of Southwestern Louisiana, Lafayette, in 1967 and 1969 , respectively, and the Ph.D. degree in electrical engineering from New Mexico State University, Las Cruces, in 1974

He is currently a Professor and Department Head of the Electrical and Computer Engineering Department, University of Louisiana, where he has been a Faculty Member for 25 years. Previously, he served two years on the faculty in the Electrical Engineering Department, Northern Arizona University, Flagstaff. He has published and presented over 60 papers and articles in the computer control and communications systems areas. He was a Senior Engineer for the Philco Ford Corporation, Houston, TX, where he conducted telecommunications research for NASA at the Johnson Space Center, Houston, TX. He has presented several industrial short courses and serves as a consultant for the telecommunications industry. $\mathrm{He}$ is recognized as a forensic engineering expert.

Dr. Henry is a registered Professional Engineer in the state of Louisiana. 